\title{
La restauration écologique du fleuve Rhône sous le double regard du sociologue et de l'écologue
}

\author{
Carole Barthélémy ${ }^{1}$, Yves Souchon ${ }^{2}$ \\ 1 Sociologue, IRD / Université de Provence, Centre Saint-Charles, Laboratoire Population, Environnement, Développement, \\ UMR151, 13331 Marseille cedex 3, France \\ 2 Écologue, CEMAGREF, UR Biologie des écosystèmes aquatiques, Laboratoire d’hydroécologie quantitative, CP 220, \\ 69336 Lyon cedex 09, France
}

\begin{abstract}
Il est assez commun que dans un processus de politique publique donnant large place à la concertation, l'objet initial soit déplacé. Il en est ainsi de ce cas d'étude qui, partant de la question des débits réservés, aboutit à celle de la lutte contre les inondations. Pour saisir cette trajectoire tracée aussi bien par la résistance du milieu aquatique que par les interactions humaines que celui-ci construit, il a fallu le regard croisé de l'écologue et du sociologue. L'hybridation est réussie, mais son efficacité tient aussi à une mise en critique des « utopies gestionnaires et scientifiques » qui voudraient un alignement pur et simple des différents types de savoirs et une heuristique propre à la dynamique des apprentissages sociaux. Asymétries, contradictions et ambivalences sont toujours là et la question de l'efficacité environnementale d'une gouvernance vertueuse reste posée.
\end{abstract}

La Rédaction

\begin{abstract}
Mots-clés :
restauration

écologique ;

concertations ;

hydrosystème fluvial ;

écologie et

sociologie ;

Rhône

Résumé - Acté en 1998, le Programme décennal de restauration hydraulique et écologique du Rhône s'est fixé comme objectif principal d'augmenter les débits réservés dans les bras court-circuités du fleuve. Cet objectif était initialement pensé comme une action de restauration écologique de milieux naturels fortement perturbés. Un processus concomitant de concertations a plusieurs fois déplacé l'intérêt initial vers des projets d'élaboration d'un territoire fluvial, puis d'un outil de gestion contre les crues. L'approche interdisciplinaire appliquée, entre sociologie et écologie, interroge la capacité d'une telle action environnementale à aboutir à des résultats hybrides, relevant à la fois de l'amélioration d'un écosystème fluvial et d'un processus de gouvernance.
\end{abstract}

\section{Keywords:}

ecological

restoration;

consultation;

fluvial hydrosystem; ecology and

sociology;

Rhone river

\begin{abstract}
A sociologist's and an ecologist's critical view of the Rhone river restoration programme. A ten-year physical and ecological restoration programme was defined for the Rhone River in 1998. Initially, one of its main objectives was to increase the minimum flow in several by-passed sections, with the clear goal of improving the fluvial habitat and the general ecological functioning of the river. A complex consultation process which brought together the hydropower industry, regional civil organisations, and local civil representatives was adopted to define more precisely the local measures to be taken. In the course of this process, the programme underwent a progressive change from purely ecological to more socially motivated outcomes such as improving amenity values and flood defence. We studied how increasing the minimum flow is a topic that can or cannot be discussed by the actors during the consultation process. For example, there is less discussion about the ecological value than about the economic value of the restoration programme between the hydropower industry and the local elected members. But, the various actors readily debate with the scientists about floods and sedimentary transport, linked with local knowledge. We applied a multidisciplinary approach, involving sociology and ecological sciences, to question the restoration programme and to analyse its hybrid results in terms of both the ecological and amenity values and at the same time to understand the governance process.
\end{abstract}

Auteur correspondant : C. Barthélémy, carole.barthelemy@univ-provence.fr 
Les cours d'eau et leur lit majeur ont depuis longtemps été aménagés pour différents usages, comme la production hydroélectrique, l'irrigation, la navigation, le gain de terres arables ou la lutte contre les inondations. Les aménagements ont produit des simplifications du tracé du lit ${ }^{1}$ et des réductions de surface des zones alluviales. En Suisse, par exemple, où $98 \%$ des ruisseaux ont été drainés, la surface de ces zones alluviales représentait $3 \%$ de la surface du pays avant 1950 et elle n'est plus aujourd'hui que de $0,25 \%$ (Zaugg Stern, 2006). Il s'en est suivi une perte nette de quantité d'habitats pour les flores et les faunes terrestres et aquatiques, une diminution de qualité de l'habitat par homogénéisation de la structure physique et une diminution de la connectivité entre habitats, réduisant les échanges de flux de matière et d'organismes dans les trois dimensions : longitudinale, latérale et verticale, des hydrosystèmes (Amoros et Bornette, 2002 ; Nedelcu et al., 2007). L'érosion de la biodiversité et la diminution de la résilience de l'hydrosystème en sont la conséquence directe (Gunderson et Holling, 2002; Décamps, 2007). Dans le même temps, plusieurs épisodes catastrophiques de crues dévastatrices (par exemple : Loire, 1980; Rhône, 1993 et 2003 ; Rhin, 1999) ont fait prendre conscience des limites des approches sécuritaires des années 1950, faisant appel à des aménagements lourds, produisant parfois des effets contraires à ceux recherchés en matière de réduction de l'aléa et de la vulnérabilité du risque inondation (Maridet et al., 1996). C'est sur ce terreau, enrichi par les réflexions de la recherche en hydroécologie des années 1970 (PIREN grands fleuves ${ }^{2}$ ), que sont nés progressivement de nouveaux concepts de gestion plus durable des fleuves, qui trouvent un début de traduction opérationnelle dans des plans de restauration ${ }^{3}$ contemporains. L'un d'entre eux, intitulé Programme décennal de restauration hydraulique et écologique du Rhône, a été initié en 1998 (Olivier et al., 2003 ; Souchon, 2004).

D'abord aménagé au XIX ${ }^{e}$ siècle pour les besoins de la navigation (Bethemont, 1997; Bravard, 1987), le Rhône a ensuite été équipé au $\mathrm{XX}^{\mathrm{e}}$ siècle par dix-neuf ouvrages hydroélectriques, lui valant l'appellation de «fleuve automatisé » pour la production énergétique production confiée à la Compagnie nationale du Rhône (CNR), concessionnaire du fleuve depuis 1934. L'enjeu de la réhabilitation se situe au niveau des secteurs du

\footnotetext{
1 Chenalisation, rectification, curage.

2 Programmes de recherche interdisciplinaire en environnement pilotés par le CNRS.

${ }^{3}$ Le vocabulaire précis dans le domaine du génie écologique voudrait que l'on réserve le terme de réhabilitation à des actions de corrections partielles du milieu physique et celui de restauration à des corrections qui visent au retour à un état structurel et fonctionnel des milieux avant leur altération. N'ignorant pas ce distinguo sémantique, nous employons quand même ici le terme de restauration dans une acception grand public, tel qu'il figure dans les textes de cadrage administratif de l'exemple proposé.
}

lit historique, ou «vieux » Rhône, court-circuités par les ouvrages et ne recevant plus qu'une infime partie du débit encore appelée " débit réservé » (par exemple, 10 à $20 \mathrm{~m}^{3} / \mathrm{s}$ pour un module de $1000 \mathrm{~m}^{3} / \mathrm{s}$ ), ainsi que certains débits de crue, lorsque le débit du fleuve dépasse le débit $\mathrm{d}$ 'armement des usines. Les contrastes hydrologiques y sont exacerbés par rapport au régime naturel. Les actions de restauration concernent :

- la restructuration morphologique des bras secondaires, les lônes, obstrués ou perchés par rapport au lit principal, suite à l'incision du chenal principal ${ }^{4}$;

- des plantations végétales (Henry et Amoros, 1995);

- la réactivation de l'érosion locale, actuellement bloquée par des structures artificielles (protection de berges, digues, épis «Girardon ») [Bravard et al., 2005]; - l'augmentation du débit réservé.

C'est cette dernière action, mise en œuvre assez tôt dans le cadre du programme décennal, qui fait l'objet de notre propos ${ }^{5}$. En effet, cette thématique de débit réservé, compromis entre l'usage énergétique et la préservation des fonctionnalités écologiques des cours d'eau, a été stimulée en France dans les années 1980, notamment à la suite de la parution de la loi de 1984 relative à la pêche en eau douce. Plusieurs travaux scientifiques ont permis de construire des modèles qui prédisent quantitativement l'évolution de l'habitat des organismes aquatiques en fonction du débit et qui sont mobilisés dans le cadre d'une aide à la décision (Bovee, 1982 ; Souchon et al., 1989 ; Pouilly, 1994 ; Lamouroux et Souchon, 2002). Si l'augmentation des débits réservés repose sur une expertise d'ordre écologique, elle est appelée à être discutée par un ensemble d'acteurs agissant distinctement autour du fleuve. En effet, dans le cadre du programme décennal, l'élévation du débit réservé n'était pas envisagée comme une action écologique univoque, mais devait, au contraire, s'inscrire dans des dynamiques socioéconomiques de revalorisation du fleuve. Des concertations à différentes échelles ont ainsi été organisées, afin d'inciter à la constitution de syndicats intercommunaux devant, à terme, valoriser les milieux fluviaux une fois restaurés. Diverses manières d'appréhender les débits réservés pouvaient ainsi être étudiées, depuis leur élaboration scientifique en modèle prédictif jusqu'à leur mise en œuvre technique à l'échelle locale, après avoir été discutés par des acteurs issus de multiples domaines, tels que celui de l'aménagement du

\footnotetext{
${ }^{4}$ Cette intervention nécessite un entretien régulier ultérieur, compte tenu de l'altération de la dynamique fluviale, liée à l'écrêtage d'une partie des crues.

${ }^{5}$ Cette recherche a été entreprise dans le cadre d'un postdoctorat réalisé au sein du Cemagref (Laboratoire d'hydroécologie quantitative [Lyon] et UMR G-EAU [Montpellier]), sous la direction d'Yves Souchon et de Patrice Garin, entre septembre 2004 et février 2006. Ce travail a fait l'objet d'une communication lors du colloque «Environnement et politique(s) », CERTOP (CNRS), Toulouse, 25 et 26 juin 2007 (Barthélémy, 2007).
} 
fleuve, du militantisme environnemental ou de la gestion locale. Du fait de cette diversité d'appropriations sociales, nous avions l'opportunité de porter un double regard, de sociologue et d'écologue, sur un processus de restauration dans un grand cours d'eau.

Une confrontation analogue avait nourri un colloque intitulé « Recréer la nature », qui s'était tenu au marais d'Orx en mai 1994, où étaient intervenus des sociologues - un numéro spécial de la revue Natures Sciences Sociétés en a rendu compte en 1995. Pour les écologues, il s'agit de s'interroger sur l'intérêt et les limites des actions de restauration écologique rencontrant un vif intérêt à partir des années 1980. Barnaud (1995) montre que, jusqu'aux années 1970, la gestion de la nature relève majoritairement de la mise en réserve, alors qu'à partir des années 1980, elle est plutôt associée à l'idée de la réparation. Pour la grande majorité des écologues, ces exercices constituent « une opportunité extraordinaire d'expérimenter en vraie grandeur et de traiter de la complexité des systèmes écologiques » (ibid., p. 42). Pour d'autres, il s'agit d'une « mission curative » dans le cadre de laquelle l'écologue peut être assimilé à un «médecin de la nature ». Quant aux plus sceptiques, ils soulignent l'ambiguïté des résultats obtenus, l'incertitude de la réactivité de l'écosystème et l'évaluation difficile de la restauration.

Fabiani (1995) apporte également des éléments intéressants quant à une approche sociologique de l'écologie de la restauration. Il montre notamment comment cette pratique est dépendante des acteurs sociaux impliqués dans les politiques gestionnaires de la nature, car l'idée de la réparation n'est pas socialement appropriée, a contrario de la mise en réserve, qui susciterait plus d'adhésion (ibid., p. 88). Un lien est tissé entre les scientifiques et les gestionnaires des espaces naturels dans l'élaboration de nouvelles normes et de réglementations d'usage des milieux restaurés.

Dans le cadre de la recherche entreprise sur la restauration écologique du Rhône, le dialogue interdisciplinaire s'est basé sur la notion de "parcours », c'est-à-dire la manière dont le programme et ses objectifs tendent à se transformer, en fonction des conditions sociopolitiques et économiques dans lesquelles il s'inscrit et en fonction d'échelles de traitement différentes, affectant directement les rapports entre la production scientifique et l'ensemble des acteurs sociaux concernés. En effet, l'expérience des écologues investis dans ce programme, depuis quelques années, aboutissait à un sentiment d'incertitude quant à la pérennité des actions entreprises. Produisant un outil d'aide à la décision éprouvé et garant, selon eux, d'une restauration écologique nécessaire autour du Rhône, ils soulignaient un certain flottement dans sa conduite. L'articulation avec l'approche sociologique s'est nouée autour de cette situation d'hésitation. De fait, cette approche s'est intéressée à la conduite non consensuelle de ce programme de restauration de la nature en privilégiant deux

\section{Encadré. L'enquête sociologique}

Dans le cadre de l'enquête sociologique, trois catégories de données ont été sollicitées. La première concerne les documents administratifs relatifs à la conduite du Programme décennal de restauration hydraulique et écologique du Rhône : rapports, comptes rendus de réunions, échanges de courriels, qui ont fait l'objet d'une analyse. La seconde concerne une enquête qualitative. Afin de recueillir les discours des acteurs participant ou ayant participé au programme, 45 entretiens semi-directifs ont été réalisés entre septembre 2004 et décembre 2005. Les représentants des organismes publics (11), les scientifiques (5) et les membres d'associations de protection de la nature (4) ont, pour la majorité, été rencontrés à Lyon. Deux bras court-circuités en cours de restauration ont été choisis dans le Rhône médian, afin de rencontrer des chargés de mission des collectivités locales (8) et des élus locaux (17). Le troisième type de données concerne des observations et des comptes rendus de concertations.

démarches. La première a consisté à suivre le parcours du programme à travers l'étude des interactions entre les multiples acteurs concernés et leurs mises en scène lors des exercices de concertation (Billaud, 2002; Mormont et al., 2006). La seconde démarche, sollicitant plus particulièrement le dialogue avec l'écologie, a étudié les débits réservés, à quel moment ils font l'objet d'études puis de discussions, comment ils peuvent cristalliser certains conflits ou, au contraire, susciter un certain consensus. Pour suivre un tel parcours, trois parties structurent notre propos. La première relate l'avènement et la mise en place du programme de restauration autour du fleuve Rhône. La seconde s'intéresse à des rapports sociaux conflictuels quant à l'augmentation des débits réservés, pour clore sur une situation où les débits réservés, au contraire, ne font pas l'objet des débats, déplacés sur un autre enjeu environnemental.

\section{Le Programme décennal de restauration hydraulique et écologique du Rhône : entre ingénierie écologique et concertations}

L'enquête sociologique réalisée par entretiens auprès de quarante-cinq personnes, ainsi que la lecture des archives administratives du programme, ont permis d'en retracer l'histoire ( $c f$. Encadré et Tab.). Ainsi, il résulte d'un conflit ayant opposé, à partir des années 1970, la CNR à des militants environnementalistes locaux. Ceux-ci sont réunis dans une fédération régionale de protection de la nature, à la fois militants associatifs et scientifiques spécialisés en hydrobiologie. Ils peuvent être qualifiés $\mathrm{d}^{\prime}$ « entrepreneurs écologistes », en s'inspirant du concept $d^{\prime}$ '« entrepreneurs de morale» d'Howard Becker (1985). L'entrepreneur de morale est défini comme «l'individu 
Tableau. Quelques dates-clés du parcours du Programme décennal de restauration hydraulique et écologique du Rhône.

\begin{tabular}{|c|c|}
\hline Dates & Parcours du Programme décennal de restauration hydraulique et écologique du Rhône \\
\hline 27 mai 1921 & $\begin{array}{l}\text { Loi concernant l'aménagement du Rhône de la frontière suisse à la mer. Celui-ci est lié à un triple objectif : } \\
\text { puissance hydraulique, navigation, irrigation }\end{array}$ \\
\hline 1933 & Création de la Compagnie nationale du Rhône (CNR), concessionnaire du fleuve, société d'économie mixte \\
\hline 8 avril 1946 & $\begin{array}{l}\text { Loi sur la nationalisation de l'électricité et du gaz : création d'EDF. Partenariat avec la CNR autour du } \\
\text { Rhône }\end{array}$ \\
\hline 1948 & Construction du premier barrage de Génissiat \\
\hline 1971 & Création de la Fédération Rhône-Alpes de protection de la nature (FRAPNA) \\
\hline 29 juin 1984 & $\begin{array}{l}\text { Loi relative à la pêche en eau douce et à la gestion des ressources piscicoles, imposant une norme quant aux } \\
\text { débits réservés } \\
\text { Exemption du Rhône et du Rhin }\end{array}$ \\
\hline 1986 & Construction du dernier barrage de Sault-Brenaz \\
\hline 1992 & $\begin{array}{l}\text { Plan d'action Rhône - agence de l'eau RMC, « Retrouver un fleuve vif et courant », dont la restauration des } \\
\text { tronçons court-circuités }\end{array}$ \\
\hline 1996 & SDAGE Rhône - agence de l'eau RMC, dont la restauration des tronçons court-circuités \\
\hline 1997 & Gouvernement de gauche plurielle. Abandon du projet de canal Rhin-Rhône \\
\hline 1998 & $\begin{array}{l}\text { Lettre interministérielle adressée au préfet de région } \\
\text { Mise en place du programme décennal }\end{array}$ \\
\hline 10 février 2000 & $\begin{array}{l}\text { Loi relative à la modernisation du service public de l'électricité : fin de la collaboration économique entre } \\
\text { EDF et la CNR }\end{array}$ \\
\hline 2000 & Restauration du débit réservé sur le site de Pierre-Bénite \\
\hline 2003 & $\begin{array}{l}\text { Inondations du Rhône } \\
\text { Lancement des concertations organisées par l'État et les régions }\end{array}$ \\
\hline 2003 & $\begin{array}{l}\text { La CNR devient une société anonyme d'intérêt général à actionnariat public majoritaire ; actionnaire privé } \\
\text { belge, Electrabel }\end{array}$ \\
\hline 2004 & Restauration du débit réservé sur le site du Haut-Rhône \\
\hline 2005 & Élaboration du plan Rhône \\
\hline 30 décembre 2006 & Loi sur l'eau éditant de nouvelles règles sur les débits réservés, dont le Rhône n'est plus exempté \\
\hline
\end{tabular}

qui entreprend une croisade pour la réforme des mœurs » (ibid., p. 171), avec le plus souvent des motifs humanitaires (contre l'alcoolisme, l'usage des drogues...) et concourant à l'élaboration de nouvelles lois et normes. Les entrepreneurs écologistes, en s'accordant sur une éthique environnementale, se sont également lancés dans une croisade contre les aménageurs afin de diffuser un autre rapport aux milieux naturels. Les connaissances développées à partir des années 1980 sur l'écosystème rhodanien vont constituer les armes de combat contre le projet de barrage de Loyettes, à la confluence de l'Ain, qui sera annulé comme le plus médiatisé canal Rhin-Rhône. Alors que la CNR et EDF, partenaires sur le Rhône de la production hydroélectrique, ont mobilisé leur personnel sur place, le nouveau Premier ministre, Lionel Jospin, porte un coup d'arrêt fatal au projet en supprimant la déclaration d'utilité publique, en juin 1997, répondant ainsi aux promesses électorales faites aux Verts lors de la constitution du gouvernement de gauche plurielle. Le ministère de l'Aménagement du territoire et de l'Environnement, sous la direction de Dominique Voynet, alors en réseau à l'échelle locale avec les entrepreneurs écologistes, soutient une de leurs propositions : pourquoi une partie du financement prévu pour le canal Rhin-Rhône ne pourrait-elle pas être réinvestie dans la restauration physique et écologique du fleuve? Un an après l'abandon du canal Rhin-Rhône, en juillet 1998, est instauré le programme décennal. Le militantisme environnemental a porté ses fruits, imposant à la CNR de financer à la hauteur de 5,3 millions d'euros par an une action environnementale, bien loin de sa vocation première d'aménageur et d'industriel. L'agence de l'eau du bassin Rhône-Méditerranée et Corse (RMC) financera à la même hauteur et un dernier tiers est attendu de la part des collectivités locales, pour atteindre un budget annuel de 15 millions d'euros.

Le programme décennal affichait deux objectifs prioritaires, dont seul le premier sera effectif : la restauration des débits réservés et 1'amélioration de la qualité écologique $\mathrm{du}$ fleuve pour la circulation des poissons migrateurs. Alors que la loi sur la pêche de 1984 imposait des règles en matière de débits réservés, le Rhône en était exempté ${ }^{6}$.

\footnotetext{
${ }^{6}$ La loi «pêche » de 1984 imposait que le débit réservé soit supérieur au $1 / 10^{\mathrm{e}}$ du module du cours d'eau. Le Rhône et le Rhin n'étaient pas concernés du fait de leur caractère « international».
} 
Cependant, des études avaient déjà été réalisées ${ }^{7}$, permettant une sélection de six aménagements jugés prioritaires, quant à une possible élévation des débit réservés, parmi les dix-neuf répartis le long du fleuve. En 1992, le plan d'action Rhône, programme d'action porté par l'agence de l'eau, citait quatre tronçons pour « leur emprise spatiale totale et leurs potentialités écologiques résiduelles »; dans le cadre du SDAGE (Schéma directeur d'aménagement et de gestion de l'eau) édité en 1996, cinq sites étaient considérés comme des «zones humides remarquables et aquifères d'intérêt patrimonial ». Au final, dans le cadre du programme décennal, en 1998, six sites seront jugés prioritaires. Les éléments permettant la sélection des sites relèvent d'une caractérisation écologique, prenant en compte les données physiques et biologiques du milieu, mais également de critères sociopolitiques. Ainsi, la volonté d'élus locaux de participer au programme influence la sélection. Sur les six bras court-circuités choisis, trois articulent un intérêt écologique et une dynamique politique locale, tandis que les trois autres n'ont été choisis qu'au niveau environnemental. L'articulation entre les critères «naturels » et sociopolitiques représenterait ainsi un facteur explicatif majeur dans la conduite du programme de restauration.

En effet, celui-ci repose sur des concertations organisées par des représentants de la Direction régionale de l'environnement (DIREN) et de l'agence de l'eau, conviant, à l'échelle d'un bras court-circuité, les maires des communes concernées, les représentants de la CNR et des services de $1^{\prime}$ État $^{8}$, des scientifiques ${ }^{9}$ et des membres d'associations de protection de la nature. Lors de ces concertations, sont discutés les divers aspects du programme de restauration, depuis le choix du débit restauré jusqu'à la mise en valeur du site. Le fait que les élus locaux soient déjà organisés facilite grandement le parcours du programme décennal, qui se structure en étapes plus ou moins longues, que nous allons présentement étudier.

\section{Des bras morts du fleuve à des milieux de vie : les enjeux de la territorialisation environnementale}

La durée des négociations locales, qui s'étend d'après les deux restaurations abouties en moyenne à une dizaine d'années, résulte d'un processus de requalification

\footnotetext{
7 Les hydroécologues ont été confrontés aux questions de débit réservé sur le Rhône à la fin des années 1970, au moment des études d'impact relatives aux travaux d'aménagement des derniers barrages du Haut-Rhône.

8 Direction départementale de l'équipement, Voies navigables de France / service de la navigation, Direction régionale de l'industrie, de la recherche et de l'environnement.

${ }^{9}$ CNRS, Université, Cemagref.
}

territoriale ${ }^{10}$. Parmi les six sites prioritaires, entre 1998 et 2007, deux ont fait l'objet d'une augmentation du débit réservé : en aval de Lyon, où le débit a été augmenté de $10-20 \mathrm{~m}^{3} / \mathrm{s}$ à $100 \mathrm{~m}^{3} / \mathrm{s}$ en 2000 , et sur le haut Rhône, où les débits ont été augmentés de $10-20 \mathrm{~m}^{3} / \mathrm{s}$ à $50-70 \mathrm{~m}^{3} / \mathrm{s}$ en 2004. Les bras morts, car non productifs pour l'ingénieur hydraulicien, reprennent vie lorsque l'écologue les considère comme un haut lieu de biodiversité. Ce milieu de vie doit ensuite être considéré comme tel par l'aménageur et les élus locaux dans le cadre d'un développement local ${ }^{11}$. Le territoire ainsi élaboré renvoie à la définition proposée par Denis Salles (2006, p. 24) des «territoires des prescriptions environnementales », soulignant le décalage entre « les territoires institutionnels de la représentation politique et les territoires des problèmes et de leur gestion ». Comment constituer un territoire sur la base d'un découpage physico-écologique du fleuve? C'est dans ce cadre qu'intervient la question des débits réservés, puisqu'il faut avant tout les modifier.

La modification est, en premier lieu, l'affaire des écologues, qui définissent des scénarios et des évaluations de la restauration. La démarche repose sur la méthode dite des «microhabitats", qui permet d'évaluer l'impact d'une augmentation du débit réservé sur la qualité de l'habitat pour les poissons (Pouilly et Souchon, 1990; Souchon et al., 2002). C'est un support de proposition pour des scénarios de restauration écologique, basés sur des augmentations des débits réservés (Lamouroux et al., 1999). Il existe beaucoup d'attente envers ces actions de restauration physique des cours d'eau, reposant soit, comme dans ce cas, sur une gestion différente des débits, soit, dans d'autres cas, sur des actions visant à remodeler directement la morphologie du lit majeur. Elles sont assez unanimement identifiées comme un investissement essentiel, nécessaire dans les prochaines décennies pour se rapprocher du bon état écologique prôné par la Directivecadre européenne sur les eaux (DCE, décembre 2000). Un suivi concernant la restauration du site en aval de Lyon a été effectué entre 1995 et 2004, alors qu'en 2000, le débit a été relevé à $100 \mathrm{~m}^{3} / \mathrm{s}$ (Lamouroux et al., 2006). Le suivi démontre que le fleuve a retrouvé un fonctionnement se rapprochant d'un fleuve "vif et courant ${ }^{12}$ ", avec une vitesse moyenne multipliée par cinq et des espèces piscicoles d'eau courante en plus forte abondance (hotu,

\footnotetext{
10 Le territoire est appréhendé comme " une forme particulière de découpage de l'espace plus ou moins institutionnalisé » (Alphandéry et Bergues, 2004, p. 5).

11 Création d'un syndicat intercommunal, mise en place de la gestion du site, accueil du public, valorisation culturelle (expositions, musées...).

12 Selon les termes mêmes des objectifs du programme décennal, il s'agissait non seulement de redonner une physionomie fluviale visible par un observateur depuis les berges, mais de permettre à un cortège $\mathrm{d}^{\prime}$ espèces de poissons représentatives des communautés fluviales de retrouver des conditions d'habitat hydraulique propices à leur rétablissement.
} 
spirlin, barbeau fluviatile...), dans des proportions suivant les prédictions des modèles de microhabitats. Ainsi, les écologues fournissent un outil d'aide à la décision, discuté lors des concertations locales.

Pour ce site en aval de Lyon, l'augmentation optimale du débit réservé était fixée à $100 \mathrm{~m}^{3} / \mathrm{s}$ pour un débit initial de $10-20 \mathrm{~m}^{3} / \mathrm{s}$, respectant la règle émise dans la loi «pêche" de 1984, du 1/10 du module moyen. $\mathrm{Si}$, pour les scientifiques, les élus locaux, les représentants de l'agence de l'eau et de la DIREN, cette proposition est rapidement acceptée, elle sera plus âprement discutée par les représentants de la CNR et d'EDF. Car l'augmentation des débits réservés constitue une perte énergétique et financière dont ils exigent une compensation. Pour la $\mathrm{CNR}$, une solution technique est trouvée en équipant d'une turbine le bras court-circuité, pouvant combler une partie de la perte énergétique. Mais la solution et l'acceptation des $100 \mathrm{~m}^{3} / \mathrm{s}$ ont surtout été le résultat des négociations qui se sont déplacées à l'échelle nationale. Pour certains acteurs interrogés, l'État a accepté le débit de $100 \mathrm{~m}^{3} / \mathrm{s}$ dans un contexte de préscission institutionnelle ${ }^{13}$ entre la $\mathrm{CNR}$ et $\mathrm{EDF}$, favorisant la première au détriment de la seconde, qui souhaitait un débit moindre.

Un autre exemple de rapports sociaux conflictuels autour des débits réservés concerne la restauration du site du Haut-Rhône. Si la valeur des débits n'a pas fait l'objet de discussions, le financement de leur augmentation a été au centre des débats. Les élus locaux, appuyés par le conseil régional, refusaient de financer les aspects hydrauliques du projet, représentant $51 \%$ du budget total, soit environ $18000 \mathrm{~K} €$ (Barthélémy, 2006). Les aspects hydrauliques concernaient les pertes énergétiques et les pertes de taxes hydroélectriques versées à l'État par la CNR. Ces taxes composant la «rente du Rhône ${ }^{14}$ » font l'objet d'un débat récurrent autour du fleuve. Pour les acteurs locaux, élus ou militants associatifs, la rente du Rhône ne devrait pas alimenter les caisses de l'État, mais être rendue aux collectivités locales afin de participer à la restauration du fleuve. La conduite du programme décennal révèle ainsi les tensions existantes entre l'État et les collectivités locales dans l'administration du fleuve. La réalisation sur le Haut-Rhône a été effective lorsque l'État a accepté de financer les aspects hydrauliques via la CNR et l'agence de l'eau.

Les deux exemples traités montrent que le choix des débits réservés est façonné par les intérêts divergents des acteurs et nécessite des détours économiques et politiques portant sur l'administration et l'exploitation du fleuve. Les nouveaux territoires fluviaux résultent de ces ajustements quant à la valeur écologique de la restauration, qui, d'une

\footnotetext{
13 Suite à la loi du 10 février 2000 relative à la modernisation du service public de l'électricité, EDF a cessé sa collaboration économique avec la $\mathrm{CNR}$ et a été remplacée par un acteur privé.

14 En 2003, la rente du Rhône s'élevait à 141 M€ (Compagnie nationale du Rhône, 2004).
}

manière générale, est essentiellement discutée pour son impact financier. Ce résultat tend à nuancer l'idée que les concertations seraient des lieux possibles de discussions scientifiques concernant les objets sociotechniques (Callon et al., 2001); autour des débits réservés, on parle peu d'écologie mais plutôt de faisabilité financière (Le Bart, 2003 ; Le Bourhis, 1999). Il faudra un événement naturel, les inondations, pour que puissent circuler les savoirs naturalistes, qu'ils soient experts ou profanes (Barthélémy, 2005), en déplaçant l'objet des débits réservés vers celui de la dynamique sédimentaire du fleuve.

\section{De la restauration écologique à la lutte contre les inondations : la circulation des savoirs experts et profanes}

Les inondations du Rhône de décembre 2003, de l'ordre de $11500 \mathrm{~m}^{3} / \mathrm{s}$ à Beaucaire, ont affecté deux sites prioritaires du programme décennal, localisés dans le Rhône moyen. Les maires des communes inondées ont alors appréhendé le programme, qui allait être effectif sur leurs territoires, comme une possible solution pour gérer les crues. Le souhait émis par ces élus d'ajouter à la restauration écologique un volet sécuritaire pose un certain nombre d'interrogations qui ont, pour première conséquence, de faire passer au second plan l'augmentation des débits réservés. Les bras court-circuités sont essentiellement perçus comme de potentiels champs d'expansion de crues, qui ne fonctionneraient plus comme tels, mais que la restauration pourrait rétablir : «Ce fameux plan décennal prévoit de revenir au niveau de ces aménagements, de remettre les lônes en activité, les nettoyer, les remettre dans leur état d'origine et, pour nous, c'est une très bonne chose. Car cette restauration va faciliter l'écoulement du Rhône ${ }^{15}$. » Ce point de vue évoqué par un élu local concerne le nettoyage des lônes afin de pouvoir contenir les débordements du Rhône. La restauration des lônes est effectivement une activité concomitante à l'augmentation des débits réservés; le niveau d'eau permet alors de reconnecter au fleuve ces bras qui ne l'étaient plus et de renaturer ces milieux naturels jugés remarquables. Mais, pour les élus rencontrés, le problème majeur réside dans le comblement des lônes et des bras court-circuités par les sédiments. Cette représentation $\mathrm{du}$ fonctionnement du fleuve, qu'il faudrait draguer, nettoyer, curer, fait véritablement fonction de «noyau dur » de la représentation (Abric, 1994) tant elle est partagée et évoquée par les élus locaux, mais également par les riverains organisés en associations, suite aux inondations. Ces derniers ont eu l'occasion de donner leurs points de vue lors des consultations organisées entre 2004 et 2006, à

\footnotetext{
15 Extrait d'un entretien réalisé avec le maire d'une commune inondée en 2003 et concernée par le programme décennal.
} 
la fois par l'État et les trois régions limitrophes du Rhône. Ces consultations, dont l'objectif affiché était de mettre en place un plan d'action ${ }^{16}$ associant les riverains et les collectivités locales aux décisions de l'État, ont fonctionné comme des lieux de rencontre entre les savoirs empiriques détenus par les habitants ou les élus et les savoirs experts diffusés par des scientifiques invités à prendre la parole.

Ainsi, « le Rhône se comble » et son corollaire «il faut draguer le fleuve » résultent d'un ensemble de savoirs empiriques. Ces derniers relèvent, tout d'abord, de ce que les élus et les riverains voient : les piles de pont ou les lônes qui se comblent deviennent des témoins infaillibles de leur raisonnement, que l'on montre à l'enquêteur lors de l'interview. Le suivi des inondations sur le terrain est aussi évoqué, l'élu connaît le cheminement du fleuve en période de crue, les lônes qu'il faudrait restaurer. De plus, le comblement entraînerait des crues plus rapides et plus importantes : à la crue "normale » qui inonde par l'aval et doucement, s'est substituée la crue « anormale », violente et imprévisible. La référence au passé, en sollicitant la mémoire locale ou des cartes datant de la fin du XIXe siècle, participe de cette catégorisation. Enfin, l'état de comblement est en général associé à une défaillance administrative de la CNR, qui aurait laissé ces bras morts à l'abandon, sans aucun entretien, aggravant de fait les inondations. Ce positionnement très contestataire à l'égard de la CNR de la part de certains riverains ou élus locaux s'est visiblement atténué au fil des réunions, notamment en prenant connaissance des aspects réglementaires : l'interdiction de toute extraction de matériaux dans le lit mineur des fleuves depuis la loi sur l'eau de 1992 et les compétences administratives de la $\mathrm{CNR}$, à qui il incombe de ne pas «aggraver la ligne d'eau » dans le cadre de ses équipements. Mais ce sont surtout les connaissances diffusées par les géomorphologues invités lors des consultations qui ont participé à l'évolution des débats. Ainsi, pour ces derniers, le dragage n'est pas indispensable, puisque les crues permettent aux sédiments grossiers, stockés dans le lit mineur, de s'évacuer. Par contre, les sédiments fins présents dans les marges alluviales ont tendance à s'accroître et à restreindre de manière significative l'espace fluvial (Bravard et al., 2005). Le projet d'ingénierie écologique consiste en l'arasement de certaines digues transversales, afin que le fleuve puisse éroder et évacuer les matériaux fins accumulés sur ses marges. Ce projet présenté comme un possible compromis entre la restauration écologique et la prévention contre les crues a fortement séduit les acteurs locaux. Il peut être analysé comme une rencontre autour de la représentation du dragage : technicisé pour les profanes, naturel pour les scientifiques, l'essentiel étant que les matériaux soient évacués.

\footnotetext{
16 Le plan Rhône, «Un projet de développement durable », a
} été acté en 2005.
Cependant, une telle perspective suscite certaines retenues de la part d'autres scientifiques, notamment du domaine de l'hydrobiologie. L'ordre de grandeur des volumes d'eau et de sédiments concernés n'est peut-être pas à la hauteur des phénomènes à corriger. Il pourrait également y avoir des impacts écologiques et écotoxicologiques à court terme lors des travaux. Certains hydrobiologistes associent la prise en compte des inondations à une dérive dangereuse du programme décennal, qui s'éloignerait trop de son objectif initial, fondamentalement écologique.

\section{Conclusion : une mise en perspective à l'échelle internationale}

Une telle distinction opérée entre restauration écologique et lutte contre les inondations est assez représentative d'approches très sectorielles, caractéristiques de l'Europe, où l'occupation humaine est ancienne et où sont multipliées les structures de gestion de différents usages (Hugues et Rood, 2003). On note néanmoins, dans certains pays, une évolution vers des approches plus intégrées, qui tendent à instaurer des pratiques plus respectueuses des principes de fonctionnement des plaines alluviales (Décamps, 1996). En Suisse, par exemple, il s'agit, en articulant les objectifs sécuritaires et environnementaux, de favoriser les zones d'expansion des cours d'eau (Nedelcu et al., 2007). Les gestions les plus holistiques se rencontrent en Australie et en Afrique du Sud, où des démarches concertées à dire d'expert définissent des objectifs de débits ciblés, entre autres, sur l'entretien des lits et le renouvellement des substrats, le maintien $\mathrm{d}$ 'habitats viables pour la faune et la flore aquatique, l'attrait hydraulique en période migratoire (Arthington et Pusey, 2003 ; King et al., 2003). Elles font également une large place à des suivis, mis en œuvre pour corriger si nécessaire les prescriptions initiales.

Ces expériences plus ou moins abouties d'articulation entre restauration écologique et lutte contre les inondations s'accompagnent également de nouvelles réflexions, quant à la mise en place d'une gestion adéquate, dont les principes d' "adaptive comanagement » (Moller et al., 2004; Olsson et al., 2004) et de "social learning » (Pahl-Wostl et al., 2008) représentent, actuellement, une tendance forte, notamment dans la gestion des cours d'eau ${ }^{17}$. Ces démarches envisagent les rapports entre la nature et les sociétés à l'instar de systèmes socioécologiques capables d'adaptation et de résilience dans une perspective de durabilité. Pour accompagner les changements affectant les systèmes, la gestion relèverait d'exercices de concertation,

\footnotetext{
17 Voir à ce propos le dossier coordonné par C. Pahl-Wostl, E. Mostert, D. Tàbara, "Social Learning in Water Resources Management », Ecology and Society, 13, 1 (http://www. ecologyandsociety.org/vol13/iss1).
} 
de participation et d'apprentissages mutuels pour coconstruire des politiques à l'interaction des connaissances expertes et profanes. La recherche présentée dans le cadre de cet article apporte quelques éléments de réflexion à l'égard de cet engouement contemporain pour la gestion co-construite des milieux aquatiques. On peut se réjouir de l'aspect concertatif de ce type de gestion, au nom d'une nouvelle manière de gouverner, rendant compte du passage d'une démocratie représentative et/ou délégative à une démocratie technique et/ou dialogique, qui serait plus à même de résoudre les problèmes environnementaux (Callon et al., 2001). L'exemple traité tend à montrer que la circulation des savoirs n'est pas effective de manière uniforme autour des objets techniques et scientifiques. Ainsi, la modélisation des débits réservés reste, quant à sa valeur écologique, l'affaire des scientifiques, tandis que les transports sédimentaires sont l'objet de représentations et de connaissances profanes. Mais on peut également adopter un point de vue opposé, faisant référence aux faiblesses et aux lenteurs des réalisations dans le domaine de l'eau. Des travaux émergent concernant les relations entre les processus de concertation et leur efficacité en termes de gains environnementaux (Salles, 2006). Dans cette perspective, les procédures, leur conduite et leurs analyses occuperaient une place disproportionnée par rapport aux objectifs écologiques des programmes d'action. La politique de l'eau en France, à l'heure de la directive-cadre européenne, illustre parfaitement cette tension : comment atteindre le bon état écologique des cours d'eau d'ici 2015, tout en valorisant l'implication des acteurs locaux, quand on connaît le délai imparti à la réalisation d'une action participative? Est-il encore nécessaire de se concerter autour des bras court-circuités du Rhône, puisque la dernière loi sur l'eau du 10 décembre 2006 impose, sur tous les cours d'eau sans exception, des règles en matière de débits réservés ${ }^{18}$ ? $S^{\prime} i l$ n'est pas question de conclure en basculant dans l'une ou l'autre de ce qui ressemble parfois à des « utopies gestionnaires », il nous paraît opportun de souligner l'intérêt de l'expérience interdisciplinaire entreprise. Certes classique dans sa forme, celle-ci génère une démarche scientifique intermédiaire, en mettant au centre de son questionnement les contradictions et les ambivalences de la gestion de l'eau. Parce que celle-ci ne relève jamais de manière tranchée d'une concertation idéale et systématisée ni d'un contrôle étatique formel et parce que les milieux naturels répondent diversement aux exercices de restauration, elle rend pertinent le dialogue entre

\footnotetext{
18 La loi du 30 décembre 2006, dite LEMA (Loi sur l'eau et les milieux aquatiques), impose de nouvelles règles en matière de débits : elle reprend les grandes lignes de la loi de 1984, généralise le débit réservé minimum à la valeur du $1 / 10^{\mathrm{e}} \mathrm{du}$ module interannuel avec effectivité en 2014, tout en introduisant une possibilité de modulation au $1 / 20^{\mathrm{e}}$. Les valeurs pour les cours d'eau de plus de $80 \mathrm{~m}^{3} / \mathrm{s}$ de module sont respectivement de $1 / 20^{\mathrm{e}}$ et $1 / 40^{\mathrm{e}}$.
}

sociologie et écologie. Un tel dialogue permet d'épouser un mouvement de balancier qui, sans chercher à systématiser les interactions nature-société, donne plutôt à voir les contours malléables des actions environnementales.

\section{Remerciements}

Ce travail a été effectué dans le cadre d'un postdoctorat de 18 mois financé par le Cemagref, impulsé par Philippe Duchène. Nous tenons à remercier l'ensemble des personnes enquêtées et les membres du comité de pilotage ayant encadré ce travail : Gabrielle Bouleau, Patrice Garin, André Micoud, Audrey Richard-Ferroudji. Les membres du Laboratoire d'hydroécologie quantitative de Lyon, et plus particulièrement André Chandesris, sont également à remercier.

\section{Références}

Abric, J-C., 1994. Pratiques sociales et représentations, Paris, PUF.

Alphandéry, P., Bergues, M., 2004. Territoires en questions : pratiques des lieux, usages d'un mot, Territoires en questions, Ethnologie française, XXXIV, 1, 5-12.

Amoros, C., Bornette, G., 2002. Connectivity and biocomplexity in waterbodies of riverine floodplains, Freshwater Biology, 47, 4, 761-776.

Arthington, A.H., Pusey, B.J., 2003. Flow restoration and protection in Australian rivers, River Research and Applications, 19, 5-6, 377-395.

Barnaud, G., 1995. À l'interface de la pratique et de la théorie : l'écologie de la restauration, Natures Sciences Sociétés, 3, horssérie Recréer la nature, 36-49.

Barthélémy, C., 2005. Les savoirs locaux, entre connaissances et reconnaissance, Vertigo, 6, 1 (http://vertigo.revues.org/ index2997.html).

Barthélémy, C., 2006. De la restauration écologique à la concertation: des territoires fluviaux en devenir? Analyse sociologique du Programme décennal de restauration hydraulique et écologique du Rhône. Rapport Cemagref, URM G-EAU, Montpellier / Laboratoire d'hydroécologie quantitative, Lyon.

Barthélémy, C., 2007. La recherche de l'efficacité environnementale modifie-t-elle l'action publique? La restauration écologique du fleuve Rhône à l'épreuve de la concertation. Communication au colloque Environnement et politique(s), organisé par le CR 23 (Environnement et développement durable) de l'AISLF et le CERTOP (CNRS), Toulouse, 25 et 26 juin.

Becker, H.S., 1985. Outsiders : études de sociologie de la déviance, Paris, Métailié.

Bethemont, J., 1997. Le Rhône entre Nation et Région, Revue de géographie de Lyon, 72, 1, 67-75.

Billaud, J.-P., 2002. Concertation et contractualisation : une mise à l'épreuve sociale et technique au cœur des enjeux de la ruralité, in Billaud, J.-P., (Ed.), Environnement et gestion des territoires : l'expérience agri-environnementale française, Paris, La Documentation française, 297-317. 
Bovee, K.D., 1982. A Guide to Stream Habitat Analysis Using the Instream Flow Incremental Methodology, Fort Collins (Co), US. Fish and Wildlife Service, Office of Biological Services.

Bravard, J.-P., 1987. Le Rhône, du Léman à Lyon, Lyon, La Manufacture.

Bravard, J.-P., Collilieux, G., Moiroud, C., 2005. L'évolution des marges le long des Vieux-Rhône : premiers résultats du diagnostic à Montélimar, Les Sédiments du Rhône : grands enjeux, premières réponses, $2^{e}$ journée thématique de la zone atelier Bassin du Rhône, Valence, 10 juin 2005, Villeurbanne, GRAIE, 86-93.

Callon, M., Lascoumes, P., Barthe, Y., 2001. Agir dans un monde incertain : essai sur la démocratie technique, Paris, Le Seuil.

Compagnie nationale du Rhône, 2004. Décembre 2003, une crue historique. Dossier "La CNR, son rôle et ses obligations".

Décamps, H., 1996. The renewal of floodplain forests along rivers: a landscape perspective, Verhandlungen Internationale Vereinigung für theoretische und angewandte Limnologie, 26, 1, 35-59.

Décamps, H., 2007. La vulnérabilité des systèmes socioécologiques aux événements extrêmes : exposition, sensibilité, résilience, Natures Sciences Sociétés, 15, 1, 48-52.

Fabiani, J.-L., 1995. Les recréateurs de nature. Enjeu et justification d'une pratique paradoxale, Natures Sciences Sociétés, 3, hors-série Recréer la nature, 84-92.

Gunderson, L.H., Holling, C.S., 2002. Panarchy, Understanding Transformations in Human and Natural Systems, Washington (DC), Island Press.

Henry, C.P., Amoros, C., 1995. Restoration ecology of riverine wetlands, II. An example in a former channel of the Rhône river, Environmental Management, 19, 6, 903-913.

Hughes, F.M.R., Rood, S.B., 2003. Allocation of river flows for restoration of floodplain forest ecosystems: A review of approaches and their applicability in Europe, Environmental Management, 32, 1, 12-33.

King, J., Brown, C., Sabet, H., 2003. A scenario-based holistic approach to environmental flow assessment for rivers, River Research and Applications, 19, 5-6, 619-639.

Lamouroux, N., Doutriaux, É., Terrier, C., Zylberblat, M., 1999. Modélisation des impacts de la gestion des débits réservés du Rhône sur les peuplements piscicoles, Bulletin français de la pêche et de la pisciculture, 352, 45-61.

Lamouroux, N., Souchon, Y., 2002. Simple predictions of instream habitat model outputs for fish habitat guilds in large streams, Freshwater Biology, 47, 8, 1 531-1 542.

Lamouroux, N., Olivier J.-M., Capra, H., Zylberblat, M., Chandesris, A., Roger, P., 2006. Fish community changes after minimum flow increase: Testing quantitative predictions in the Rhône River at Pierre-Bénite, France, Freshwater Biology, 51, 9, 1 730-1 743.

Le Bart, C., 2003. Les nouveaux espaces de la citoyenneté, la citoyenneté locale, Les Nouvelles Dimensions de la citoyenneté, Cahiers français, 316, 58-62.

Le Bourhis, J.-P. 1999. Les commissions locales de l'eau : quelques remarques sur la mise en œuvre d'un dispositif de planification négocié, in Marié, M., Larcena, D., Dérioz, P. (Eds), Cultures, usages et stratégies de l'eau en Méditerranée occidentale : tensions, conflits et régulation, Paris, L'Harmattan, 521-533.

Reçu le 17 mars 2008. Accepté le 5 février 2009.
Maridet, L., Piégay, H., Gilard, O., Thévenet, A., 1996. L'embâcle de bois en rivière : un bienfait écologique? Un facteur de risques naturels?, La Houille blanche, 5, 32-38.

Moller, H., Berkes, F., Lyver, P., Kislalioglu, M., 2004. Combining science and traditional ecological knowledge : monitoring populations for co-management, Ecology and Society, 9, 3.

Mormont, M., Mougenot, C., Dasnoy, C., 2006. La participation composante du développement durable : quatre études de cas, Vertigo , 7, 2 (http ://vertigo.revues.org/index2346.html).

Nedelcu, M., Hainard, F., Guex, D., Gobat, J-M., 2007. Fonctions multiples, contraintes multiples : les défis du nouveau paradigme de gestion des zones alluviales suisses, Natures Sciences Sociétés, 15, 1, 3-12.

Olivier, J.-M., Lamouroux, N., Amoros, C., Carrel, G., 2003. Scientific assessment of the "Rhône river rehabilitation project". The second International Symposium on the Management of Large rivers for Fisheries, Sustaining Livelihoods and Biodiversity in the New Millenium, 11th-4th February, Phnom Penh, Kingdom of Cambodia.

Olsson, P., Folke, P., Berkes, F., 2004. Adaptive comanagement for building resilience in social-ecological systems, Environmental Management, 34, 1, 75-90.

Pahl-Wostl, C., Mostert, E., Tàbara, D., 2008. The growing importance of social learning in water resources management and sustainability science, Ecology and Society, 13, 1 (http://www.ecologyandsociety.org/vol13/iss1/art24/).

Pouilly, M.,1994. Relations entre l'habitat physique et les poissons des zones à cyprinidés rhéophiles dans trois cours d'eau du bassin rhodanien : vers une simulation de la capacité d'accueil pour les peuplements. Thèse de doctorat, Université Claude Bernard Lyon 1, Lyon.

Pouilly, M., Souchon, Y., 1990. Simulation des capacités d'habitat potentiel des poissons. Schéma départemental de vocation piscicole du fleuve Rhône, tronçon court-circuité de Montélimar, Cemagref, Délégation de bassin RMC, Lyon.

Salles, D., 2006. Les Défis de l'environnement : démocratie et efficacité, Paris, Syllepse.

Souchon, Y., 2004. The Rhône river: hydromorphological and ecological rehabilitation of a heavily man-used hydrosystem, Assessment and Provision of Environmental Flows in Mediterranean Watercourses: Basic Concepts, Methodologies and Emerging Practice, IUCN Mediterranean Office, 3-11.

Souchon, Y., Trocherie, F., Fragnoud, E., Lacombe, C., 1989. Les modèles numériques des microhabitats des poissons : application et nouveaux développements, Revue des sciences de l'eau, 2, 4, 807-830.

Souchon, Y., Andriamahefa, H., Breil, P., Albert, M.-B., Capra, H., Lamouroux, N., 2002. Vers de nouveaux outils pour l'aide à la gestion des hydrosystèmes : couplage des recherches physiques et biologiques sur les cours d'eau, Natures Sciences Sociétés, 10, Suppl. 1, 26-41.

Zaugg Stern, C., 2006. De la décision d'expert au dialogue sur les risques, Eawag news 61f, 12-14. 